Journal of

Synchrotron

Radiation

ISSN 0909-0495

Received 6 June 2010

Accepted 3 February 2011

\title{
Revealing low-dose radiation damage using single-crystal spectroscopy
}

\author{
Robin L. Owen, ${ }^{a *}$ Briony A. Yorke, ${ }^{b}$ James A. Gowdy ${ }^{b}$ and Arwen R. Pearson ${ }^{b}$ \\ ${ }^{a}$ Diamond Light Source Ltd, Diamond House, Harwell Science and Innovation Campus, \\ Didcot, OX11 ODE, UK, and ${ }^{\mathbf{b}}$ Astbury Centre for Structural Molecular Biology, University \\ of Leeds, Leeds, UK. E-mail: robin.owen@diamond.ac.uk
}

\begin{abstract}
The structural information and functional insight obtained from X-ray crystallography can be enhanced by the use of complementary spectroscopies. Here the information that can be obtained from spectroscopic methods commonly used in conjunction with X-ray crystallography and best-practice single-crystal UV-Vis absorption data collection are briefly reviewed. Using data collected with the in situ system at the Swiss Light Source, the time and dose scales of low-dose X-ray-induced radiation damage and solvated electron generation in metalloproteins at $100 \mathrm{~K}$ are investigated. The effect of dose rate on these scales is also discussed.
\end{abstract}

Keywords: macromolecular crystallography; single-crystal microspectrophotometry; radiation damage; myoglobin; cytochrome $c$.

\section{Introduction}

Structural biology aims to provide detailed insight into biological function at the molecular and atomic level. However, in extrapolating from the static average structures obtained from X-ray crystallography, care must be taken to ensure that radiation damage has not affected the structure determined. While the effect of high absorbed X-ray doses is often readily observed in the form of a loss of diffracted intensity, radiation damage begins to accrue as soon as the $\mathrm{X}$-ray shutter is opened and the absorbed dose is still small (Garman, 2010). This low-dose damage is not immediately apparent from examination of diffraction images or data processing statistics, but may only be revealed in, for example, a lack of anomalous signal or in the derived electron density map (Holton, 2009). It is therefore important to assess the time and dose scales over which the initial stages of radiation damage occur. A greater understanding of these, and of the underlying physical processes involved, will aid the design of data collection strategies which result in electron density maps with minimal radiation damage contamination.

On-line single-crystal spectrometers are capable of recording X-ray-induced changes in the crystal during irradiation and have proven invaluable in tracking rapid X-rayinduced changes (Pearson et al., 2004). The availability of dose-dependent spectral information can guide data collection strategies, enabling the determination of undamaged structures and identification of X-ray-induced intermediates that would have otherwise been impossible to obtain (Berglund $e t$ al., 2002; Schlichting et al., 2000). Single-crystal spectrometers are now available at synchrotron sources worldwide providing in situ access to a range of spectroscopic windows. These include, but are not limited to, the facilities at the European Synchrotron Radiation Facility (ESRF) (Bourgeois et al., 2002; Royant et al., 2007; Carpentier et al., 2007; McGeehan et al., 2009), National Synchrotron Light Source (NSLS) (Orville et al., 2009), the on-axis system at the Swiss Light Source (SLS) (Owen et al., 2009) and the 4DX system at the Advanced Photon Source (APS) (Pearson et al., 2007). A more detailed recent review of available facilities can be found by Pearson \& Owen (2009).

The role on-line spectroscopy can play in following X-rayinduced changes has proven particularly crucial for metalloproteins (Berglund et al., 2002; Schlichting et al., 2000; Yano et al., 2005; Pearson et al., 2007; Hough et al., 2008). Metals are often involved in catalytic, regulatory and structural roles, and precise knowledge of the redox state of a metal in a structure is crucial as redox properties are central to function. During an X-ray experiment, however, metal centres combine the undesirable properties of a high X-ray cross section (photoelectric effect and Compton scattering) and a susceptibility to X-ray-induced change. Spectroscopic studies have revealed the half-life of oxidized species in an X-ray beam to be an order of magnitude less than that of the diffracting power of a crystal, emphasizing the need for complementary methods to monitor these changes. These include [spectroscopic technique used, dose limit (citation)]: disordering of selenomethionine sidechains [XANES, 2 MGy (Holton, 2007)], reduction of copper nitrate reductase [UVVis, XAS, 1.5 MGy (Hough et al., 2008)], cleavage of anomalous scatterers [XAS, 0.5 MGy (Oliéric et al., 2007)], photoreduction of putidaredoxin [XAS, 0.3 MGy (Corbett $e t$ al., 2007)] and reduction of Met-Mb [UV-Vis, 0.21 MGy (Beitlich et al., 2007)]. 
It is important to note that the optical and X-ray spectroscopies available provide insight into a wide range of physical properties and the spectroscopy to be exploited should be chosen with reference to the problem in hand. UV-Vis absorption, for example, requires a chromophore and provides information on oxidation states and ligand-to-metal charge transfers, while Raman spectroscopy probes the vibrational motions of atoms (Carey, 2006;

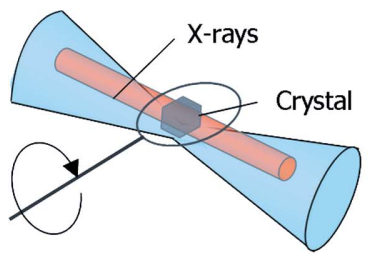

(a)

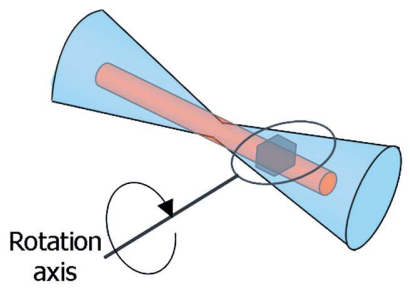

(b)

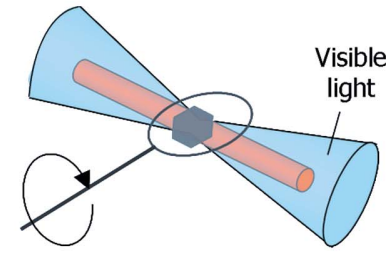

(c)

Figure 1

Schematic of an on-axis system showing (a) sample rotation axis and X-rays well aligned with respect to one another but misaligned with respect to visible light, $(b) \mathrm{X}$-rays and visible light well aligned but sample rotation axis misaligned, and $(c)$ good alignment of all components.

McGeehan et al., 2007; Carpentier et al.,

2007). X-ray spectroscopies such as XANES (X-ray absorption near-edge structure) and EXAFS (extended X-ray absorption fine structure) can provide information on the oxidation state, site symmetry and coordination numbers of metal sites (Yano et al., 2005; Corbett et al., 2007; Ellis et al., 2008).

For proteins not containing a metal or chromophore, the number of spectroscopies that can be exploited for on-line monitoring of X-ray-induced changes is reduced. Vibrational spectroscopies such as Raman and IR absorption are possible, but are not well suited to high-throughput macromolecular crystallography owing to either long acquisition times (Raman) or water absorption (IR). However, in a non-highthroughput environment, single-crystal Raman spectra of very high quality can be obtained and these have proved to be extremely informative, both in identifying the nature of bound ligands or adducts and in identifying radiation-induced changes (Carey, 2006; McGeehan et al., 2007; Katona et al., 2007).

Here we present a study of the effect of dose rate on haemcentre X-ray reduction in myoglobin and cytochrome $c$ at cryo-temperatures $(100 \mathrm{~K})$. To aid and encourage researchers wishing to combine X-ray crystallography and spectroscopy, we have also included a section with some guidelines on the collection of high-quality artefact-free single-crystal UV-vis spectra $(\$ 2)$.

\section{Collection of high-quality single-crystal UV-Vis absorption spectra}

The use of UV-Vis absorption spectroscopy to establish the radiation sensitivity of a sample is dependent on the collection of high-quality artefact-free spectra. Several key points for the collection of such data are outlined below. Additional useful information on sample preparation and recording of spectra can be found by Wilmot et al. (2002).

Before the collection of spectroscopic data it is important to confirm that both the X-ray beam and the UV-Vis light path probe the same sample volume and intersect exactly with the sample rotation axis (Fig. 1). Alignment of the light path and rotation axis is best achieved with a small pinhole $(\sim 10 \mu \mathrm{m}$ diameter) carefully centred at the sample position. The amount of light transmitted by this hole (from both objectives) should be maximized via small adjustments to the position of each lens. If an on-axis geometry is used, a scintillator placed at the sample position can be used to confirm alignment of the X-ray and optical light paths [Fig. 5 of Owen et al. (2009)]. Alignment can be confirmed by the use of a standard sample such as a haem protein which exhibits distinctive and easily observable spectral changes upon reduction by X-ray irradiation and of which there are many examples in the literature.

Many ideal practices in sample preparation from X-ray diffraction also apply to single-crystal UV-Vis absorption spectroscopy. The amount of solvent surrounding the crystal should be minimized, i.e. crystals should be suspended within a thin film rather than a large volume of solvent and cryoprotectant. Optimized cryoprotection is also critical to a much greater extent than in the X-ray diffraction experiment. Ice is highly undesirable as this reduces the transparency of the sample and leads to aberrant absorption features. Surface ice can be removed, or avoided by careful orientation of the sample though this may then limit the number of suitable positions for data collection. Small changes in position over the surface of the crystal, together with rotation of the sample, can result in large spectral changes both in the form of resolution of features and a changing background level (Fig. 2). This is due to a combination of prism effects and the anisotropic nature of chromophores in single crystals where they are preferentially oriented by their packing in the lattice (Wilmot et al., 2002). An optimal orientation that closely resembles the solution spectrum can usually be found. Owing to this dependence on position and angle, if spectral changes are to be tracked during diffraction data collection then an optimal angle must be found and returned to for each UV-Vis data collection. For this reason, in particular at room temperature, it may be necessary to establish the time scales of $\mathrm{X}$-ray-induced changes on a stationary sacrificial crystal prior to diffraction data collection. The accessible rotation range for UV-Vis data collection is also limited by scattering from the loop used for crystal mounting. Loops introduce unwanted spectral features and noise (Fig. 2) and should therefore be chosen so that the focal spot of the lamp comfortably fits within the loop cross-sectional area. An ideally mounted sample will also provide sufficient space for a reference spectrum to be collected on the mother liquor or cryobuffer alone. This is particularly advantageous for crystals of high optical density as longer exposure times can be used before 


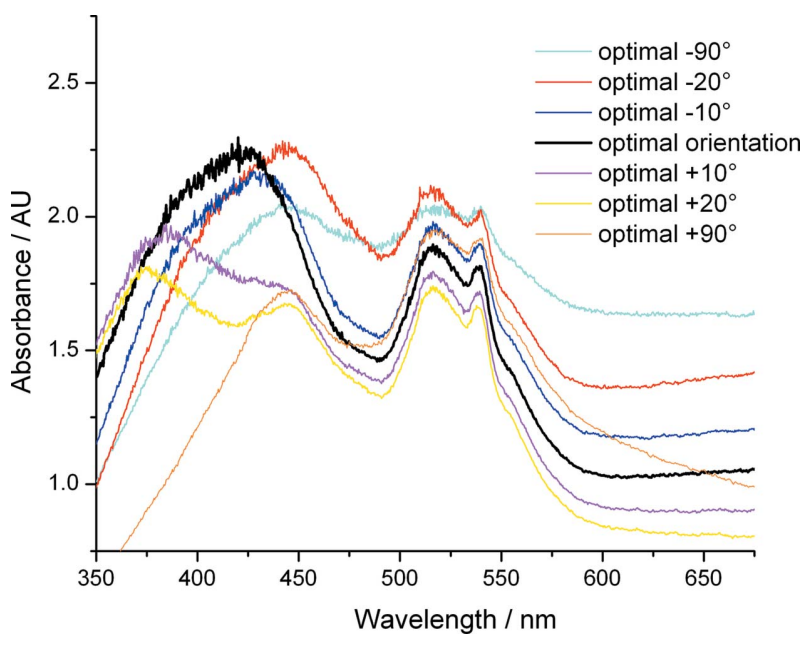

Figure 2

Horse heart cytochrome $c$ single-crystal spectra showing variations in both absorption peaks and baseline as a function of crystal orientation. The optimal orientation is obtained when the loop face is almost perpendicular to the UV-Vis light path and a facet of the crystal is in the same plane. Spectra are the average of ten $20 \mathrm{~ms}$ exposures and were collected using a 4DX single-crystal spectrometer (4DX Systems), illuminated by a balanced deuterium/halogen light source (Ocean Optics), attached to a Shamrock 163 spectrograph and Newton CCD detector (Andor Technology). For crystallization conditions, see §3.1.

lamp features saturate the detector introducing spectral artefacts.

While it is difficult, or almost impossible, to improve the spectral properties of an inherently poor or icy sample, weak spectra or spectra from crystals with a high optical density can be improved at the point of data collection by careful choice of data collection parameters. For example, the emission spectrum of the xenon light source used in the SLS microspectrophotometer is considerably stronger at longer wavelengths $(>500 \mathrm{~nm})$ than in the $350-450 \mathrm{~nm}$ region. This has the consequence that, when recording a spectrum from a sample with high optical density at low wavelength, e.g. haem, increasing the exposure time in order to produce a measurable change in absorbance for the strongly absorbing Soret band (usually located between $\sim 400$ and $450 \mathrm{~nm}$ ) results in saturation of the detector above $500 \mathrm{~nm}$, obliterating the weaker $\alpha$ - and $\beta$-bands (located between 500 and $600 \mathrm{~nm}$ ). Filters can be used to equalize the illumination spectrum to avoid this problem.

\section{Materials and methods}

\subsection{Sample preparation}

Solutions of horse myoglobin (Sigma) at $20 \mathrm{mg} \mathrm{ml}^{-1}$ in $50 \%(v / v)$ 2-methyl-2,4-pentanediol (MPD) were doped with increasing amounts of sodium bromide $(0-1.0 \mathrm{M}$ in $0.1 \mathrm{M}$ steps), and solutions of bovine cytochrome $c$ (Sigma) at $20 \mathrm{mg} \mathrm{ml}^{-1}$ in $50 \%(v / v)$ MPD were doped with $\mathrm{NaCl}\left(0_{-}\right.$ $1.0 M$ in $0.1 M$ steps). To avoid any confounding elements in this study of the effects of X-ray illumination, no additional buffer components were added. The solutions were prepared from stocks such that the concentration of protein was constant in all samples. Thin films of the solutions were cryocooled in $0.2-0.5 \mathrm{~mm}$-diameter nylon $(20 \mu \mathrm{m}$ thick) loops at $100 \mathrm{~K}$ using a cryojet (Oxford Instruments). Visual inspection indicated the film thickness to be less than the thickness of the loops.

Horse cytochrome $c$ (Sigma) crystals were grown according to the method of Sanishvili et al. (1994), using drops containing $40 \mathrm{mg} \mathrm{ml}^{-1}$ cytochrome $c, 50 \mathrm{mM}$ potassium phosphate $\mathrm{pH} 6.8$ and 22\% PEG $1000(w / v)$ equilibrated against a reservoir of $30 \%(w / v)$ PEG 1000 and $50 \mathrm{mM}$ potassium phosphate $\mathrm{pH} 6.8$. Crystals $(\sim 100 \times 100 \times 300 \mu \mathrm{m})$ were soaked for $2 \mathrm{~min}$ in mother liquor containing increasing amounts of $\mathrm{NaCl}(0-0.6 M)$ or $\mathrm{Na}_{2} \mathrm{MoO}_{4}(0-0.6 M)$, harvested in nylon loops and directly cryo-cooled at $100 \mathrm{~K}$ in a cryojet without any additional cryoprotectant.

\subsection{Spectroscopic data collection}

The on-axis arrangement previously described (Owen et al., 2009) at beamline X10SA of the SLS was used for all X-ray reduction experiments. An X-ray beamsize of $50 \times 50 \mu \mathrm{m}$ was used for all experiments, while the focal spot size of the Xenon lamp at the sample was $\sim 35 \mu \mathrm{m}$.

When illuminated by X-rays, glycerol has been reported to give a broad strong visible absorption signal centred at $600 \mathrm{~nm}$, that has been assigned to the generation of solvated electrons (McGeehan et al., 2009). As myoglobin and cytochrome $c$ have absorption bands that extend into the red and near-IR regions [i.e. the $\alpha$ and $\beta$ bands are in the $500-600 \mathrm{~nm}$ region (Lippard \& Berg, 1994)], we investigated MPD as an alternative cryoprotectant for the thin-film studies. It has been reported that there is no significant change in MPD absorption over the spectral range of interest upon X-ray irradiation (McGeehan et al., 2009) (Fig. 3). On-line UV-Vis spectra were recorded over $30 \mathrm{~s}$ (53.6 spectra per second with an acquisition time of $10 \mathrm{~ms}$ per spectrum) and the X-ray shutter was opened a short time $(<4 \mathrm{~s})$ after the UV-Vis spectra accumulation began (X-ray energy $12.4 \mathrm{keV}$ with a flux of $1.1 \times 10^{11}$ photons $\mathrm{s}^{-1}$ ), allowing time courses to be fitted over $>25 \mathrm{~s}$ of $\mathrm{X}$-ray exposure.

In order to probe the effect of changing the mother liquor $\mathrm{X}$-ray absorption cross section on the rate of haem centre reduction, on-line UV-Vis spectra were recorded, as described above, from myoglobin and cytochrome $c$ thin films in triplicate for each halide $\left(\mathrm{Cl}^{-}\right.$and $\left.\mathrm{Br}^{-}\right)$concentration during continual X-ray exposure as above. Thin films were used since the optical density of the cytochrome $c$ single crystals was so high that changes in the Soret band could not be accurately observed. However, to confirm similar effects were seen in crystals and in solution, the experiment was repeated using cytochrome $c$ crystals doped with $\mathrm{NaCl}$ and $\mathrm{Na}_{2} \mathrm{MoO}_{4}$, as changes in the $\alpha$ and $\beta$ bands (but not the Soret) could be directly compared with those observed in the thin films.

Finally, a second series of spectra were recorded for the myoglobin thin films doped with $\mathrm{NaBr}(0-1 M)$ at a lower flux rate of $5 \times 10^{10}$ photons $\mathrm{s}^{-1}$, maintaining the X-ray energy at $12.4 \mathrm{keV}$. 


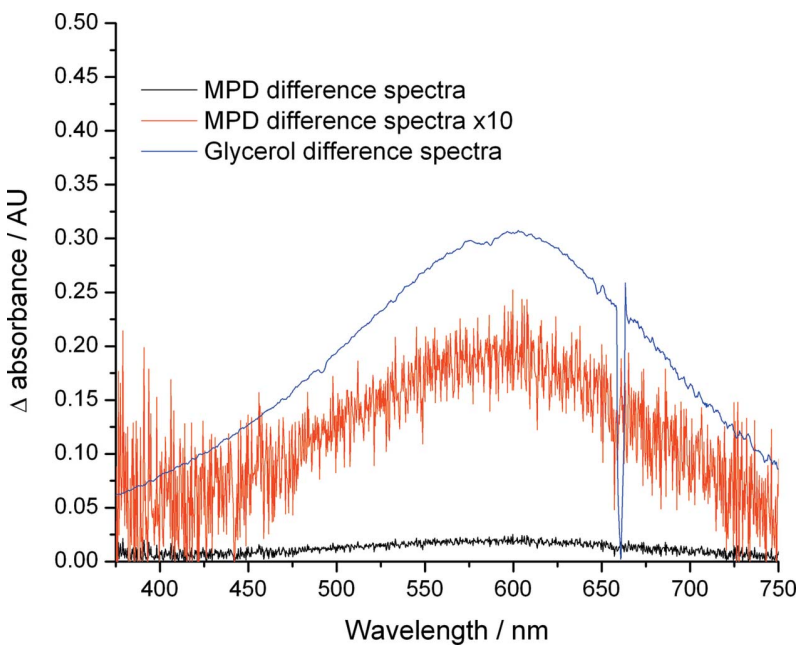

Figure 3

Difference spectra (X-ray shutter open - X-ray shutter closed) showing the appearance of a broad feature centred at $600 \mathrm{~nm}$ in solutions of $50 \%$ $(v / v)$ MPD (recorded in this study) and $40 \%(v / v)$ glycerol [recorded at the ESRF (McGeehan et al., 2009)] at $100 \mathrm{~K}$ during X-ray exposure. The discontinuity in the glycerol spectrum at $680 \mathrm{~nm}$ is a deuterium lamp artefact.

\subsection{Data analysis}

Single-wavelength time courses for the shift in the Soret band (from 413 to $426 \mathrm{~nm}$ for myoglobin and 411 to $415 \mathrm{~nm}$ for cytochrome $c)$ and the appearance of the $\alpha(557 \mathrm{~nm}$ myoglobin, $535 \mathrm{~nm}$ cytochrome $c$ ) peaks (characteristic of reduced haem) and at $600 \mathrm{~nm}$ (assigned to solvated electron formation) were extracted from the data. Kinetic parameters were determined using Gnuplot, Biokine (Biologic) and OriginPro (Origin Lab) and analysed using OriginPro. Myoglobin peaks were assigned based on spectra for myoglobin reduced by ${ }^{60} \mathrm{Co} \gamma$ radiation at $77 \mathrm{~K}$ [where myoglobin cannot undergo the conformational rearrangements that normally accompany reduction (Gasyna, 1979)]. Cytochrome $c$ peaks were assigned based on literature values (Keilin \& Slater, 1953).

Absorbed-dose calculations for the protein samples were carried out using RADDOSE (Paithankar et al., 2009), assuming a constant sample volume and a non-rotating sample.

Rate constants for myoglobin and cytochrome $c$ reduction by X-rays were determined for two features in the spectra: the shift in the Soret band and the increase in the $\alpha$-band (Fig. 4). Also fitted were the time courses for absorbance changes at $600 \mathrm{~nm}$ for both proteins in 50\% ( $/ / v)$ MPD as well as for $50 \%$ $(v / v)$ MPD alone. All data were best fitted with a double exponential, as has previously been reported for metmyoglobin X-ray reduction as a function of incident X-ray energy (Beitlich et al., 2007). This function is of the form

$$
y=A_{1} \exp \left(-k_{1} D\right)+A_{2} \exp \left(-k_{2} D\right)+y_{0}
$$

where $y$ is the absorbance of the Soret or $\alpha$-band, $k_{1}$ and $k_{2}$ are the fast and slow rates, respectively, $A_{1}$ and $A_{2}$ are the amplitudes of the two exponents, and $D$ is the absorbed dose. All rate constants were thus in units of dose $\mathrm{e}^{-1}$. The function is constrained so that the fast process described by $k_{1}$ occurs first.

\section{Results}

\subsection{Effect of cryoprotectant used on spectral changes}

In order to establish marker wavelengths representative of solvated electrons rather than of long-wavelength haem protein bands, optical spectra were collected from the cryoprotectant, MPD, alone during X-ray illumination (Fig. 3). These data are compared with spectra from $40 \%$ glycerol previously recorded at the ESRF and reported by McGeehan et al. (2009).

Although it has been reported that no distinct absorbance peak associated with solvated electrons is observed in solutions of MPD (McGeehan et al., 2009), a weak broad increase in absorbance in this region was observed when $50 \%(\mathrm{v} / \mathrm{v})$ MPD alone was exposed to X-rays. The change in absorbance of MPD at $600 \mathrm{~nm}$ is small, but is easily resolvable by the SLS microspectrophotometer.

Since the absorbance change at $600 \mathrm{~nm}$ upon X-ray illumination of MPD is so small, it does not mask the temporal evolution of haem protein $\alpha$ and $\beta$ bands between 500 and $560 \mathrm{~nm}$. MPD was therefore used as a cryoprotectant for the subsequent experiments reported here.

\subsection{X-ray-induced changes to oxidized myoglobin and cytochrome $c$}

To establish whether the rate of reduction of the haem centres of myoglobin and cytochrome $c$ during X-ray irradiation is affected by X-ray dose rate at $100 \mathrm{~K}$, in contrast to 'classical' radiation damage (loss of diffracted intensities, changes in unit-cell dimensions, disulfide bond breakages, decarboxylations, etc.) which is dose-rate independent at cryotemperatures (Garman, 2010), we monitored the rate of haem reduction during continuous $\mathrm{X}$-ray exposure in cryo-cooled thin films $(\sim 20 \mu \mathrm{m}$ thick $)$ of both proteins. To alter the dose rate we doped solutions of both proteins with increasing concentrations of $\mathrm{NaBr}$ and $\mathrm{NaCl}$. An increase in $\mathrm{NaBr}$ concentration from $0 M$ to $1 M$, for example, results in a $49 \%$ increase in the X-ray absorption cross section of the solution, with a consequent change in absorbed dose from $19 \mathrm{kGy} \mathrm{s}^{-1}$ to $28 \mathrm{kGy} \mathrm{s}^{-1}$ (for the same incident flux and exposure time, an increase in $\mathrm{NaCl}$ concentration from $0 M$ to $1 M$ corresponds to a change in absorbed dose from 16 to $26 \mathrm{kGy} \mathrm{s}^{-1}$ ). We also prepared cryo-cooled cytochrome $c$ crystals that had been soaked in artificial mother liquor containing $\mathrm{NaCl}, \mathrm{NaBr}$ and $\mathrm{Na}_{2} \mathrm{MoO}_{4}$.

The spectral changes that occur upon X-ray irradiation of both proteins are indicative of haem reduction. The Soret band is red shifted and the broad band at 530-550 nm splits into two distinct peaks [Figs. 4(a) and 4(b)]. A clear isosbestic point (indicating a two-state system) at $419.5 \mathrm{~nm}$ and $413.1 \mathrm{~nm}$ was observed in the thin-film data for myoglobin and cyto- 
(a)

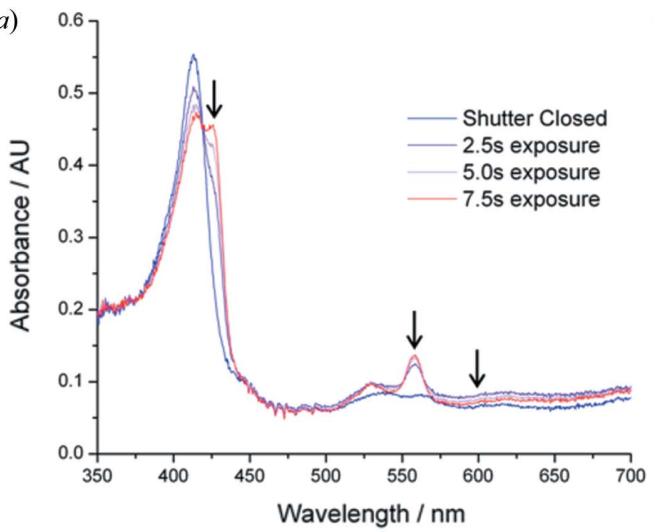

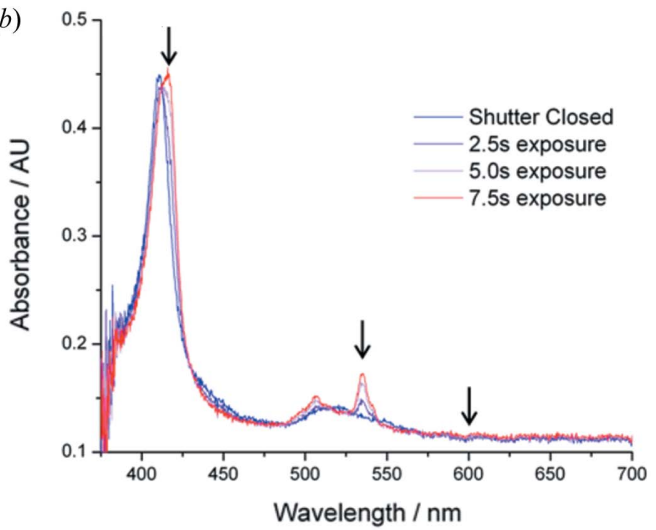

(d)

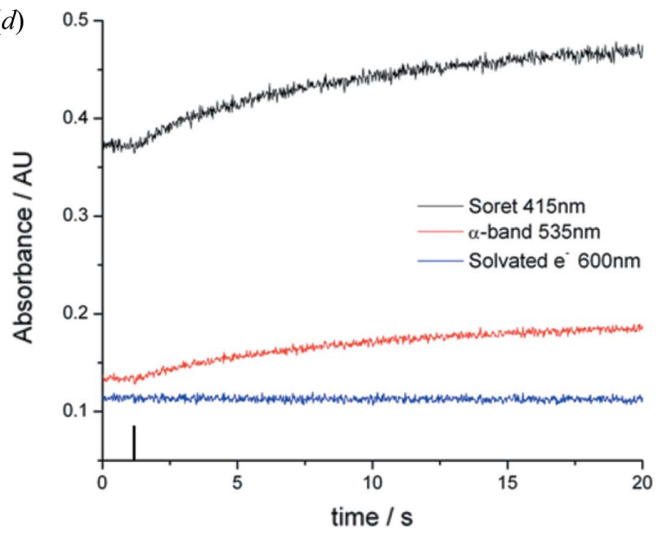

(b)

Figure 4

(c)

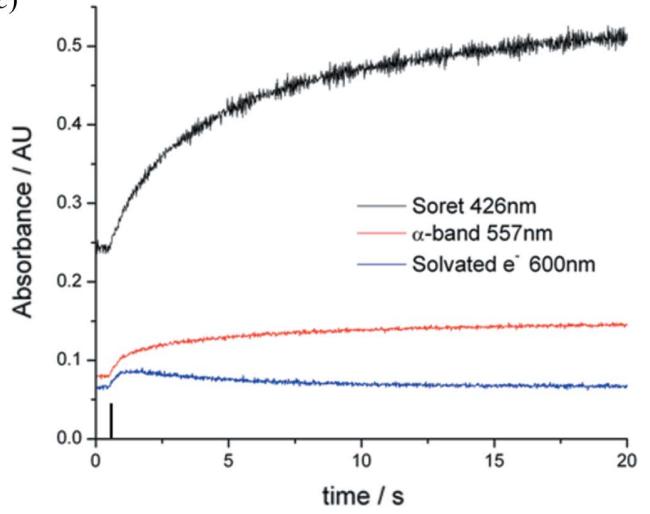

Absorption spectra of $(a)$ myoglobin and $(b)$ cytochrome $c$ thin films doped with $0.5 \mathrm{M} \mathrm{NaBr}$ before and after exposure to X-ray radiation recorded using the SLS on-axis microspectrophotometer. Several intermediate spectra are shown to highlight the isosbestic point in each system. The arrows show, from left to right, the position of the Soret and $\alpha$-bands upon X-ray reduction and the $600 \mathrm{~nm}$ feature. Sample extracted time courses for $(c)$ myoglobin and $(d)$ cytochrome $c$ showing the changes in absorbance at the three wavelengths of interest versus time (accumulated dose). The upright line on the X-axis indicates the point at which the X-ray shutter was opened; from this point the sample was continuously irradiated.

chrome $c$, respectively. Despite the presence of an isosbestic point the time courses extracted for the shift in the Soret band and the appearance of the $\alpha$-band, as well as the spectral changes at $600 \mathrm{~nm}$, show a biphasic behaviour in all samples [as previously observed by Beitlich et al. (2007) for myoglobin] indicating that two kinetically distinct processes are occurring [Figs. 4(c) and 4(d)]. The changes at $600 \mathrm{~nm}$, shown to be the $\lambda_{\text {max }}$ of solvated electrons in MPD ( $\left.\$ 4.1\right)$, were taken to be representative of solvated electron formation and decay. The extracted spectral time courses were best fit by a double exponential function and the results are summarized in Fig. 5.

The observed rate constants $k_{1}$ and $k_{2}\left(\mathrm{~Gy}^{-1}\right)$ are each similar at all three wavelengths studied and are essentially constant with absorbed dose, indicating that the X-rayinduced rapid reduction of the haem centres and the rate of solvated electron generation is independent of dose rate.

The dose at which the population of solvated electrons reaches a maximum was determined from the fit parameters $A_{1}, k_{1}, A_{2}$ and $k_{2}$ for the myoglobin thin film $600 \mathrm{~nm}$ time courses at two incident fluxes and a range of $\mathrm{NaBr}$ concentrations by differentiation of equation (1) (Fig. 6). This 'turnover dose' was found to be slightly increased in the highflux regime, but almost constant at different halide concentrations (mean value $45 \mathrm{kGy}$ ).

\section{Discussion}

Extensive studies of radiation damage by several groups have demonstrated that classical radiation damage (loss of diffracted intensities, changes in unit-cell dimensions, disulfide bond breakages, decarboxylations, etc.) is purely a function of the cumulative absorbed dose (Holton, 2009; Garman, 2010). The cumulative absorbed dose is dependent on the X-ray absorption cross section of the illuminated volume (which can include the crystal itself as well as surrounding mother liquor and the mount). A comprehensive study by Kmetko et al. (2006) showed clearly that the composition of the mother liquor, and hence the absorption cross section of the sample, does affect the rate of accumulation of classical radiation damage, and suggested that attempts to reduce the X-ray absorption cross section of the mother liquor should extend crystal life-time at $100 \mathrm{~K}$ because the dose is reduced.

Here we have investigated whether the rapid X-ray reduction of the haem centre in myoglobin and cytochrome $c$ at low cumulative X-ray doses is also independent of dose rate. Our data show clearly that the rate of accumulation of this lowdose damage is independent of dose rate at $100 \mathrm{~K}$.

A relatively small increase and subsequent decrease in absorbance was observed at $600 \mathrm{~nm}$ upon irradiation of 
(a)

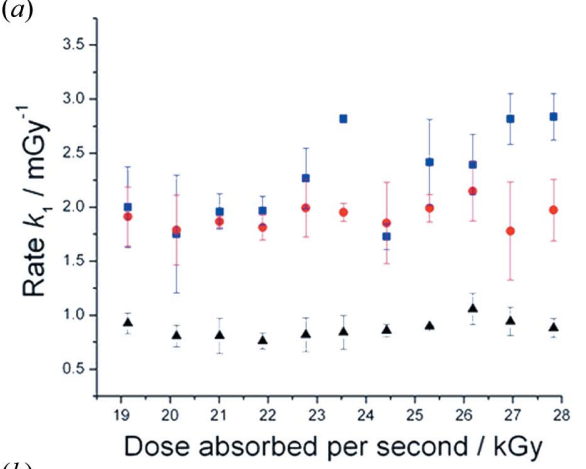

(b)

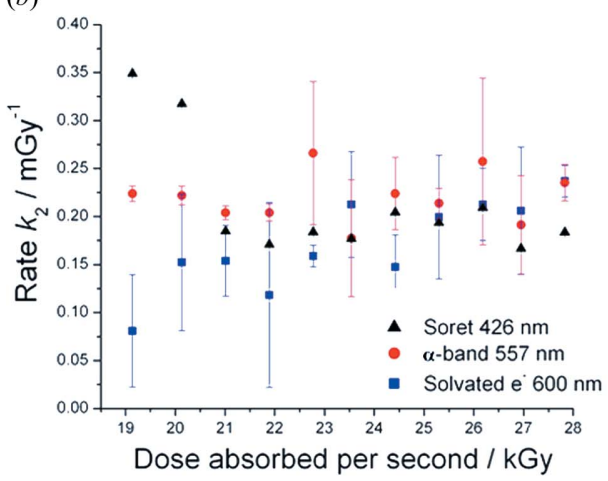

(c)

(d)
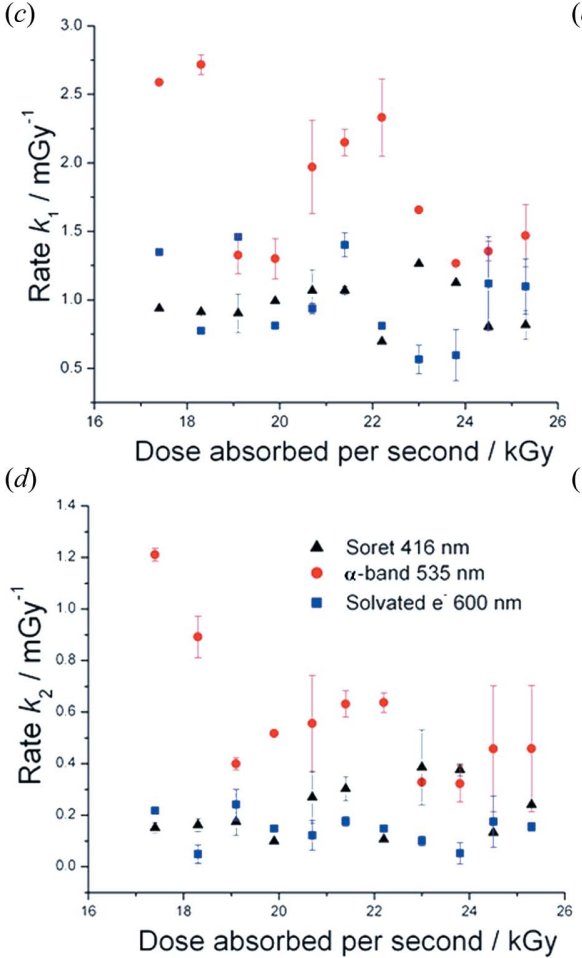
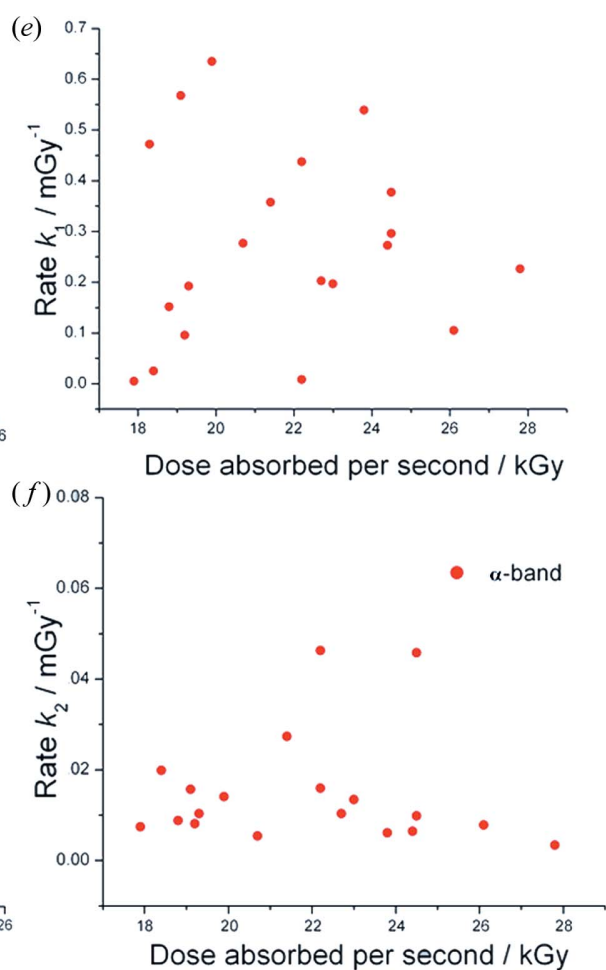

Figure 5

Rate constants for $(a, b)$ myoglobin thin films doped with increasing concentrations of $\mathrm{NaBr}(0-1.0 M$ in $0.1 M$ steps from left to right), $(c, d)$ cytochrome $c$ thin films doped with increasing concentration of $\mathrm{NaCl}$ and $(e, f)$ horse heart cytochrome $c$ crystals doped with $\mathrm{NaBr}, \mathrm{NaCl}$ and $\mathrm{Na}_{2} \mathrm{MoO}_{4} . k_{1}$ for each is shown in the top row, while $k_{2}$ is shown at the bottom. Each point is the mean value of three observations. Cytochrome $c$ crystal $k_{2}$ data have been scaled to exclude a single outlier at $0.23 \mathrm{mGy}^{-1}$. Owing to saturation effects and the difficulty in tracking the weak solvated electron signal in crystals, only the $\alpha$-band has been tracked for cytochrome $c$ crystal data.

myoglobin, cytochrome $c$ and also of MPD alone. By comparison with the better characterized absorption maximum of glycerol upon X-ray irradiation (Ershov \& Pikaev, 1968; McGeehan et al., 2009), this was attributed to solvated electrons. Interestingly this peak grows and decays with rate constants that are strikingly similar to the two rate constants required to fit the X-ray-driven reduction of the

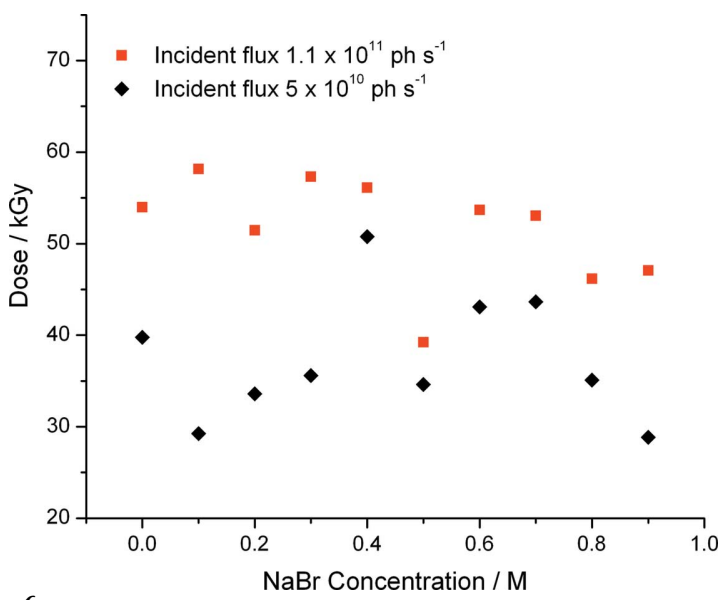

Figure 6

The absorbed dose at which the solvated electron population reaches a maximum in thin films of myoglobin (as determined from the change in absorption at $600 \mathrm{~nm}$ ) as a function of both sodium bromide concentration and incident flux rate. Each point is the average of three measurements. haem proteins reported here. Beitlich et al. (2007), who studied changes at $557 \mathrm{~nm}$ (nominally the $\alpha$ band of myoglobin, but where the contribution of the absorbance of solvated electrons can also be significant) upon X-irradiation of myoglobin, suggested that $k_{1}$ (the faster rate) was representative of the formation of solvated electrons upon X-ray illumination. They assigned the slower $k_{2}$ as representative of the haem reduction rate when the concentration of solvated electrons had reached a steady state. The similarity of the $k_{2}$ for both myoglobin and cytochrome $c$ reduction to that we observed for the decay of the $600 \mathrm{~nm}$ feature, which presumably reflects the decay to a steady-state level of solvated electrons, supports this hypothesis.

In view of this, we determined the dose at which the population of solvated electrons reaches a maximum (i.e. the maximal absorbance at $600 \mathrm{~nm}$ ), before decaying to its steady state and found that this occurs at $\sim 45 \mathrm{kGy}$. This value is a function only of the absorbed dose and not the mother liquor composition (other than in the way this composition affects the absorption cross sections) or incident flux. While this dose is somewhat smaller than the dose limits summarized in $\S 1$ for the reduction of metal centres at $100 \mathrm{~K}$ by synchrotron X-ray irradiation (0.2-2 MGy), it is comparable with dose limits observed spectroscopically during $\gamma$-radiolysis of myoglobin at relatively low dose rates [yield of $50 \%$ reduced myoglobin generated at an absorbed dose of $20 \mathrm{kGy}$ with an incident dose rate of $220 \mathrm{~Gy} \mathrm{~min}^{-1}$ (Denisov et al., 2007)]. 
Taken together, these observations suggest that reducing the absorption cross section of the crystallization mother liquor, either by transferring the crystal into a weakly absorbing mother liquor/cryoprotectant solution or by backsoaking to remove non-bound anomalous scatterers, will somewhat extend the lifetime of oxidized redox centres in the $\mathrm{X}$-ray beam. However, this gain is likely to be small, as the rate of the haem centre reduction appears to be strongly coupled to the generation of solvated electrons that, in myoglobin in MPD, reaches a maximum at an absorbed dose of only $45 \mathrm{kGy}$. With the beamline fluxes available at third-generation synchrotron sources, this can easily be reached within the time required to collect a single diffraction image.

Therefore, a composite data collection strategy in which small wedges of data are collected from several crystals (or several points on a large single crystal) remains the best, and possibly the only, approach to obtaining the structures of fully oxidized and partially oxidized species.

\section{Summary}

Complementary methods provide valuable information both on the properties of a sample and how these change upon $\mathrm{X}$-ray irradiation. These changes occur on dose scales that are much smaller than are observable through changes in the quality of the X-ray diffraction pattern. The maximum population of solvated photoelectrons in this study was reached by a dose of $\sim 45 \mathrm{kGy}$, some three orders of magnitude smaller than the dose limit based on diffracting power (Owen et al., 2006). At third-generation synchrotron sources, absorbed doses of this magnitude can be easily reached when collecting a single image, highlighting the limitations of diffraction-based methods for tracking low-dose radiation damage. The use of complementary methods during the diffraction experiment is therefore extremely important to establish what structural state the recorded diffraction data describe, and is essential for the recognition and tracking of low-dose radiation damage.

We thank Martin Fuchs, Guillaume Pompidour, Vincent Thominet and Clemens Schulze-Briese at the SLS for assistance with the microspectrophotometer set-up and data collection. We would also like to thank James Holton, Elena Kovaleva and Mike Webb for stimulating and critical discussion. BAY is supported by the WT four-year PhD programme 'The Molecular Basis of Biological Mechanisms', awarded to the Astbury Centre for Structural Molecular Biology. JAG was supported by a Biochemical Society Summer Studentship and ARP is supported by a RCUK Academic Research Fellowship.

\section{References}

Beitlich, T., Kühnel, K., Schulze-Briese, C., Shoeman, R. L. \& Schlichting, I. (2007). J. Synchrotron Rad. 14, 11-23.
Berglund, G. I., Carlsson, G. H., Smith, A. T., Szöke, H., Henriksen, A. \& Hajdu, J. (2002). Nature (London), 417, 463-468.

Bourgeois, D., Vernede, X., Adam, V., Fioravanti, E. \& Ursby, T. (2002). J. Appl. Cryst. 35, 319-326.

Carey, P. R. (2006). Annu. Rev. Phys. Chem. 57, 527-554.

Carpentier, P., Royant, A., Ohana, J. \& Bourgeois, D. (2007). J. Appl. Cryst. 40, 1113-1122.

Corbett, M. C., Latimer, M. J., Poulos, T. L., Sevrioukova, I. F., Hodgson, K. O. \& Hedman, B. (2007). Acta Cryst. D63, 951-960.

Denisov, I. G., Victoria, D. C. \& Sligar, S. G. (2007). Radiat. Phys. Chem. 76, 714-721.

Ellis, M. J., Buffey, S. G., Hough, M. A. \& Hasnain, S. S. (2008). J. Synchrotron Rad. 15, 433-439.

Ershov, B. G. \& Pikaev, A. K. (1968). Stabilization of Electrons in Low Temperature Radiolysis of Polar Systems, Radiation Chemistry, pp. 1-24. Washington: American Chemical Society.

Garman, E. F. (2010). Acta Cryst. D66, 339-351.

Gasyna, Z. (1979). Biochim. Biophys. Acta, 577, 207-216.

Holton, J. M. (2007). J. Synchrotron Rad. 14, 51-72.

Holton, J. M. (2009). J. Synchrotron Rad. 16, 133-142.

Hough, M. A., Antonyuk, S. V., Strange, R. W., Eady, R. R. \& Hasnain, S. S. (2008). J. Mol. Biol. 378, 353-361.

Katona, G., Carpentier, P., Nivière, V., Amara, P., Adam, V., Ohana, J., Tsanov, N. \& Bourgeois, D. (2007). Science, 316, 449-453.

Keilin, D. \& Slater, E. C. (1953). Br. Med. Bull. 9, 89-97.

Kmetko, J., Husseini, N. S., Naides, M., Kalinin, Y. \& Thorne, R. E. (2006). Acta Cryst. D62, 1030-1038.

Lippard, S. \& Berg, J. (1994). Principles of Bioinorganic Chemistry, pp. 283-348. Mill Valley: University Science Books.

McGeehan, J. E., Carpentier, P., Royant, A., Bourgeois, D. \& Ravelli, R. B. G. (2007). J. Synchrotron Rad. 14, 99-108.

McGeehan, J., Ravelli, R. B. G., Murray, J. W., Owen, R. L., Cipriani, F., McSweeney, S., Weik, M. \& Garman, E. F. (2009). J. Synchrotron Rad. 16, 163-172.

Oliéric, V., Ennifar, E., Meents, A., Fleurant, M., Besnard, C., Pattison, P., Schiltz, M., Schulze-Briese, C. \& Dumas, P. (2007). Acta Cryst. D63, 759-768.

Orville, A. M., Lountos, G. T., Finnegan, S., Gadda, G. \& Prabhakar, R. (2009). Biochemistry, 48, 720-728.

Owen, R. L., Pearson, A. R., Meents, A., Boehler, P., Thominet, V. \& Schulze-Briese, C. (2009). J. Synchrotron Rad. 16, 173-182.

Owen, R. L., Rudiño-Piñera, E. \& Garman, E. F. (2006). Proc. Natl Acad. Sci. USA, 103, 4912-4917.

Paithankar, K. S., Owen, R. L. \& Garman, E. F. (2009). J. Synchrotron Rad. 16, 152-162.

Pearson, A., Mozzarelli, A. \& Rossi, G. (2004). Curr. Opin. Struct. Biol. 14, 1-7.

Pearson, A. R. \& Owen, R. L. (2009). Biochem. Soc. Trans. 37, 378381.

Pearson, A. R., Pahl, R., Kovaleva, E. G., Davidson, V. L. \& Wilmot, C. M. (2007). J. Synchrotron Rad. 14, 92-98.

Royant, A., Carpentier, P., Ohana, J., McGeehan, J., Paetzold, B., Noirclerc-Savoye, M., Vernède, X., Adam, V. \& Bourgeois, D. (2007). J. Appl. Cryst. 40, 1105-1112.

Sanishvili, R. G., Margoliash, E., Westbrook, M. L., Westbrook, E. M. \& Volz, K. W. (1994). Acta Cryst. D50, 687-694.

Schlichting, I., Berendzen, J., Chu, K., Stock, A. M., Maves, S. A., Benson, D. E., Sweet, R. M., Ringe, D., Petsko, G. A. \& Sligar, S. G. (2000). Science, 287, 1615-1622.

Wilmot, C. M., Sjögren, T., Carlsson, G. H., Berglund, G. I. \& Hajdu, J. (2002). Methods Enzymol. 353, 301-318.

Yano, J., Kern, J., Irrgang, K. D., Latimer, M. J., Bergmann, U., Glatzel, P., Pushkar, Y., Biesiadka, J., Loll, B., Sauer, K., Messinger, J., Zouni, A. \& Yachandra, V. K. (2005). Proc. Natl Acad. Sci. USA, 102, 12047-12052. 